\title{
ToF-SIMS Microanalysis of Patterned Protein Surfaces
}

\author{
David G. Castner
}

National ESCA and Surface Analysis Center for Biomedical Problems, Departments of Chemical Engineering and Bioengineering, Box 351750 University of Washington, Seattle, WA 98195-1750 USA

Biomedical surface analysis has undergone significant and numerous advances in the past 30 years in terms of improved instrumentation, introduction of new techniques, development of sophisticated data analysis methods, and the increasing complexity of samples analyzed. Comprehensive analysis of surfaces and surface immobilized biomolecules (peptides, proteins, DNA, etc.) with modern surface analysis instrumentation provides an unprecedented level of detail about the immobilization process and the structure of the immobilized biomolecules. Results from x-ray photoelectron spectroscopy (XPS or ESCA), time-of-flight secondary ion mass spectrometry (ToF-SIMS), near edge x-ray absorption fine structure (NEXAFS), surface plasmon resonance (SPR) biosensing, atomic force microscopy, and sum frequency generation (SFG) vibrational spectroscopy provide important information about the attachment, orientation, conformation, etc. of biomolecules. Due to the complexity and volume of data generated by these surface analysis techniques, multivariate data analysis methods are often required to fully analyze the data. As patterned bioactive structures are of increasing interest for numerous biomedical applications, this talk will present the latest experimental results on ToF-SIMS microanalysis of patterned protein surfaces.

ToF-SIMS can provide the chemically specific images needed to characterize these patterned bioactive surfaces. In one study two affinity ligands (biotin and chloroalkane) were photolithographically patterned onto a glass slide coated with a poly(ethylene glycol) (PEG) based polymer [1]. Exposure of this surface to single and mixed solutions of streptavidin and HaloTag® resulted in spontaneous formation of high-fidelity protein patterned surfaces (see Figure 1). Fluorescence and ToF-SIMS images after protein immobilization showed high specificity of the target proteins to their respective surface patterned ligands. In addition, ToF-SIMS detected the presence of non-specific adsorption of bovine serum albumin, a masking protein present in excess in the protein solutions, onto the patterned surfaces. Protein amino acid composition, surface coverage, density and orientation are important parameters that determine the relative ToF-SIMS fragmentation pattern yields. ToF-SIMS amino acid-derived ion fragment yields summed to produce surface images can reliably determine which patterned surface regions contain bound proteins, but do not readily discriminate between different co-planar protein regions. Principal component analysis (PCA) of these ToF-SIMS data, however, improves discrimination of ions specific to each protein, facilitating surface pattern discrimination based on protein type. Also, ToF-SIMS imaging detected regions where residue from incompletely removed UV-exposed photoresist was present [2]. The presence of the photoresist residue was the likely cause of reduced streptavidin fluorescence intensity observed from those regions.

In another study patterns of antibodies oriented in opposite directions (i.e., the Fab region pointing away from the polymer surface or facing downward towards the polymer surface) were characterized with ToF-SIMS [3]. Images based on the sum of all amino acid peak intensities provided information about the relative concentration of the antibodies in each region. Ratios of the amino acid 
peak intensities that were present in different concentrations in the Fab and Fc regions of the antibody provided information about the orientation of the surface bound antibodies.

\section{References}

[1] M. Dubey, K. Emoto, H. Takahashi, D.G. Castner and D.W. Grainger, Advanced Functional Materials 19 (2009) 3046.

[2] M. Dubey, K. Emoto, F. Cheng, L.J. Gamble, H. Takahashi, D.W. Grainger and D.G. Castner, Surface and Interface Analysis 41 (2009) 645.

[3] F. Liu, M. Dubey, H. Takahashi, D.G. Castner and D.W. Grainger, Analytical Chemistry (2009) submitted.

[4] This research was supported by grants EB-002027 and EB-001473 from the National Institutes of Health.

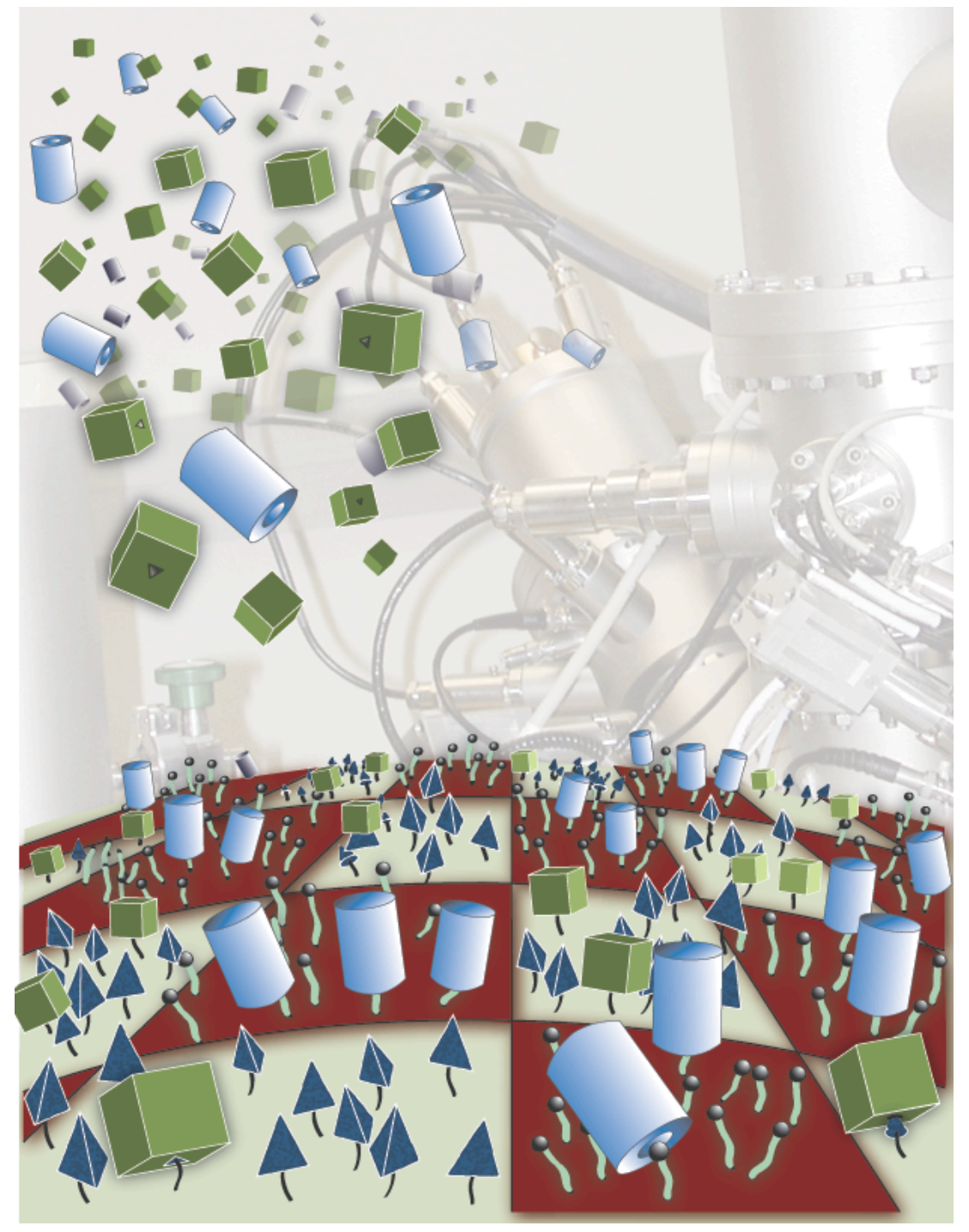

FIG. 1. Schematic cartoon depicting the assembly of a patterned protein surface from a mixed protein solution. 